1 Hacettepe Journal of Mathematics and Statistics

$\bigcap$ Volume 46 (6) (2017), $1077-1091$

\title{
Existence and qualitative properties of solutions of a second order mixed type impulsive differential equation with piecewise constant arguments
}

\author{
Gizem. S. OZTEPE*†
}

\begin{abstract}
We prove the existence and uniqueness of the solutions of a second order mixed type impulsive differential equation with piecewise constant arguments. Moreover, we study oscillation, non-oscillation and periodicity of the solutions.
\end{abstract}

Keywords: Existence of solutions, Oscillation, Non-oscillation, Periodicity, Impulsive differential equation, Piecewise constant argument.

2000 AMS Classification: 34K11, 34K13, 34K45.

Received : 28.11.2016 Accepted : 17.01.2017 Doi : 10.15672/HJMS.2017.431

\section{Introduction}

In the recent years impulsive differential equations with piecewise constant arguments attract attention of many mathematicians and numerous papers have been published on this class of equations. Most of the publications are devoted to first order differential equations. Among them note the papers [1]-[4] are on oscillation and periodicity problems. However, qualitative behaviours of second order impulsive differential equations and second order differential equations with piecewise constant arguments are the subject of many investigations while second order impulsive differential equations with piecewise constant arguments are not. Our paper is probably one of the first publications concerned with qualitative behaviour problems of second order impulsive delay differential equations with piecewise constant arguments. Now let us give an quick overview on the existing literature of the subject. Firstly, start with second order impulsive differential equations:

In [5], Huang study the oscillation and non-oscillation for the second order linear impulsive differential equation $u^{\prime \prime}=-p(t) u$ where $p(t)$ is an impulsive function defined by $p(t)=\sum_{n=1}^{\infty} a_{n} \delta\left(t-t_{n}\right)$ and establish a necessary and sufficient condition for oscillation (or non-oscillation) of the equation $u^{\prime \prime}=-p(t) u$. In 1999, Berezansky

${ }^{*}$ Department of Mathematics, Faculty of Sciences, Ankara University, 06100, Ankara, Turkey Email: gseyhan@ankara.edu.tr

${ }^{\dagger}$ Corresponding Author. 
and Braverman establish the following properties of a second order impulsive differential equation: non-oscillation of the differential equation and the corresponding differential inequality, positiveness of the fundamental function and the existence of a solution of a generalized Riccati inequality. Explicit conditions for non-oscillation and oscillation and comparison theorems are presented [6]. The paper [7] is devoted to the study of the oscillatory behaviour of a type of extensively studied second-order nonlinear delay differential equations with impulses. They show in some examples that, even though some delay differential equations without impulses are non-oscillatory, they may become oscillatory if some impulses are added to them. That is, in some cases, impulses play a dominating part in causing the oscillations of equations. In 2004, Yan obtain an explicit necessary and sufficient condition for all bounded solutions to be oscillatory by using the comparison theorem on bounded oscillation of the impulsive differential equation with the corresponding non impulsive differential equation[8]. Luo and Shen use the associated Riccati techniques and the equivalence transformation to discuss the oscillation and the non-oscillation of the second order linear ordinary differential equation with impulses in [9]. In [10], the authors give a non-oscillation criterion for second-order half-linear equation with periodic coefficients under fixed moments of impulse actions. The method is based on the existence of positive solutions of the related Riccati equation and a recently obtained comparison principle. In the special case when the equation becomes impulsive Hill equation new oscillation criteria are also obtained. Ozbekler and Zafer [11] consider the nonlinear impulsive equation of the form

$$
\begin{gathered}
\left(r(t) x^{\prime}\right)^{\prime}+\sum_{k=1}^{n} q_{k}(t) \phi_{k}(x)=f(t), t \neq \theta_{i} ; \\
\Delta r(t) x^{\prime}+\sum_{k=1}^{n} q_{i, k} \phi_{k}(x)=f_{i}, t=\theta_{i},
\end{gathered}
$$

where $\Delta g(t)=g\left(t^{+}\right)-g\left(t^{-}\right)$with $g\left(t^{ \pm}\right)=\lim _{\tau \rightarrow t^{ \pm}} g(\tau)$. The authors give new oscillation criteria for this equation. The method is based on the existence of a nonprincipal solution of a related second order impulsive differential equation

$$
\begin{aligned}
\left(r(t) z^{\prime}\right)^{\prime}+Q(t) z & =0, t \neq \theta_{i} \\
\Delta r(t) z^{\prime}+Q_{i} z & =0, t=\theta_{i},
\end{aligned}
$$

where $Q(t)=\sum_{k=1}^{n} q_{k}(t)$ and $Q_{i}=\sum_{k=1}^{n} q_{i, k}$.

Now let us continue with papers on second order differential equations with piecewise constant arguments:

A few investigations are available in the current literature on the modelling and analysis of the behaviour of physical systems in dynamics with governing equations of second order piecewise constant differential equations. In 1988, Leung [12] study the steady state response of a linear mechanical system in which the forcing function is represented by a linear combination of known functions that can be continuous or piecewise constant. The solution of the system is assumed in the form of a linear combination of the given functions with unknown coefficients. For a piecewise constant forcing function, the response at the discrete points of time is obtained. In 1994, Dai and Singh [13] present the solutions of several second order differential equations representing motions of a springmass system disturbed by a piecewise constant force in the form of $f([t])$ or $f(x([t]))$. Then, in 1997, they replace the variable $t$ of the continuous function $f(t)$ in equation

$$
m x^{\prime \prime}+c x^{\prime}+k x=f(t), t>0
$$


with the piecewise constant variable $\frac{[N t]}{N}$ such that a piecewise constant system is obtained with the governing equation

$$
m x^{\prime \prime}+c x^{\prime}+k x=g\left(\frac{[N t]}{N}\right),[N t] / N \leq t \leq([N t]+1) / N
$$

and they show that this replacing is a good approximation to the given function $f(t)$ with argument $t$, if the parameter $N$ is sufficiently large [14]. Here, Eq.(1.1) is the form of an equation which describes vibratory motion associated with simple and complicated dynamic systems in engineering applications can often be simplified to the motion of spring-mass system with one degree of freedom where the time dependent function $f(t)$ is known and express the continuous external force acting on the spring mass system. Later, in 1998, again Dai and Singh [15] use the piecewise constant technique which they have investigated in their earlier works [13], [14] to obtain approximate and numerical solutions of the driven Froude pendulum. By the application of the piecewise constant technique they prove that approximate solutions for the non-linear system can be derived and a numerical simulation for the oscillations of the driven Froude pendulum may be conveniently performed on a computer. They also examine the oscillatory behaviour of the pendulum on the basis of numerical solutions obtained. In 1999, Wiener and Lakshmikantham [16] consider the equation of the type

$$
x^{\prime \prime}(t)-a^{2} x(t)=b x([t-1]), b \neq 0 .
$$

In addition to forming the explicit solutions of Eq.(1.2) they find some sufficient conditions on oscillatory, non-oscillatory as well as periodic solutions with period 3. In [17], Seifert consider functional differential equations with piecewise constant arguments and give conditions under which such equations with almost periodic time dependence have unique almost periodic solutions, and for certain autonomous cases, they obtain certain stability results and also conditions for chaotic behaviour of solutions. The aim of the paper [18] is to show that a bounded solution of a second-order differential equation with piecewise constant argument of the form $(x(t)+p x(t-1))^{\prime \prime}=q x([t])+f(t)$ is almost automorphic. In [19] and [20] almost and pseudo-almost periodic solutions for the second-order neutral differential equations with piecewise constant argument are investigated. Green's function for second order differential equations with piecewise constant arguments is studied in [21] and [22]. Dads and Lhachimi give necessary and sufficient conditions in order to ensure the existence and uniqueness of pseudo almost periodic solutions in [23]. The existence of a solution, existence of almost and quasi-periodic solutions and the spectrum containment of almost periodic solution for a class of second-order functional differential equations with piecewise constant arguments are investigated in [24], [25] and [26], respectively. In [27], we consider the second order differential equation with piecewise constant mixed arguments

$$
x^{\prime \prime}(t)-a^{2} x(t)=b x([t-1])+c x([t])+d x([t+1])
$$

where $a, b, c, d \in \mathbb{R}-\{0\}$ and [.] denotes the greatest integer function. We prove the existence and uniqueness of solutions of this equation, state the necessary and sufficient conditions that make the zero solution is a global attractor and derive some conditions under which it has oscillatory, non-oscillatory and k periodic solutions.

All these have made us motivated to study the qualitative aspects of the second order mixed type impulsive differential equation with piecewise constant arguments. 
In this paper, we consider the following second order mixed type impulsive differential equation with piecewise constant arguments:

$$
\begin{aligned}
x^{\prime \prime}(t)-a^{2} x(t) & =b x([t-1])+c x([t])+d x([t+1]), t \neq n, t \geq 0, \\
\Delta x^{\prime}(n) & =\alpha x^{\prime}(n), t=n, n \in \mathbb{Z}^{+},
\end{aligned}
$$

where $a, b, c, d$ and $\alpha$ are real constants which are different from zero, $\mathbb{Z}^{+}=\{1,2, \ldots\}$, $\Delta x^{\prime}(n)=x^{\prime}\left(n^{+}\right)-x^{\prime}\left(n^{-}\right)$such that $x^{\prime}\left(n^{+}\right)=\lim _{t \rightarrow n^{+}} x^{\prime}(t)$ and $x^{\prime}\left(n^{-}\right)=\lim _{t \rightarrow n^{-}} x^{\prime}(t)$, and [.] denotes the greatest integer function.

This paper is organised as below:

The existence and uniqueness of solutions of Eq.(1.3)-(1.4) is studied in section 2; section 3 is devoted to the main results such as oscillation, non-oscillation and periodicity.

It should be emphasized that the results obtained for Eq.(1.3)-(1.4) coincide with the results for equations without impulses in [27] when we take $\alpha=0$ in (1.4).

Now, let us give the definition for a solution and the oscillation of a solution

1.1. Definition. A function $x(t)$ defined on $\Omega:\{-1\} \cup[0, \infty)$ is said to be a solution of the Eq.(1.3)-(1.4) if it satisfies the following conditions:

(i) $x: \Omega \rightarrow \mathbb{R}$ is continuous on $[0, \infty)$,

(ii) $x^{\prime}(t)$ exists and is continuous on $[0, \infty)$ with the possible exception of the points $[t] \in \mathbb{Z}^{+}$

(iii) At the points $[t] \in \mathbb{Z}^{+}, x^{\prime}(t)$ is right continuous and has left hand side limits,

(iv) $x^{\prime \prime}(t)$ exists at each point $t \in[0, \infty)$ with the possible exception of the points $[t] \in \mathbb{Z}^{+}$where one-sided derivatives exist,

(v) $x(t)$ satisfies $(1.3)$ for any $t \in[0, \infty)$ with the possible exception of the points $[t] \in \mathbb{Z}^{+}$

(vi) $x^{\prime}(t)$ satisfies (1.4) for every $[t] \in \mathbb{Z}^{+}$.

1.2. Definition. A solution $x(t)$ of Eq.(1.3)-(1.4) is said to be oscillatory if $x(t)$ has arbitrarily large zeros, otherwise it is called non-oscillatory.

\section{Existence and Uniqueness of Solutions}

This section includes theorems which insures that Eq.(1.3)-(1.4) has a solution and with the initial conditions it is unique.

Note that along this section it is assumed that $\alpha \neq 1$ and $d \neq \frac{a^{2}}{\cosh a-1}$.

2.1. Theorem. Eq.(1.3)-(1.4) has a solution on $[0, \infty)$.

Proof. Denote

$$
x_{n}(t)=x_{n}(n+s)=\lambda^{n} v(s)
$$

is a solution of Eq.(1.3)-(1.4) on the interval $[n, n+1)$, where $n \in \mathbb{Z}^{+}, \lambda$ is a constant, $0 \leq$ $s<1$ and $v:[0,1) \rightarrow \mathbb{R}$ is a continuous function and consider the initial conditions

$$
v(0)=1, \quad v(1)=\lambda .
$$

On the other hand, for $n \leq t<n+1$, Eq.(1.3) is written as

$$
\frac{d^{2} x_{n}(t)}{d t^{2}}-a^{2} x_{n}(t)=b x_{n}(n-1)+c x_{n}(n)+d x_{n}(n+1) .
$$

In view of (2.1) and (2.2),

$$
x_{n}(n-1)=\lambda^{n-1}, x_{n}(n)=\lambda^{n}, x_{n}(n+1)=\lambda^{n+1} \text { and } \frac{d^{2} x_{n}(t)}{d t^{2}}=\lambda^{n} \frac{d^{2} v}{d s^{2}} .
$$


Substituting (2.4) in (2.3), we get

$$
v^{\prime \prime}-a^{2} v=b \lambda^{-1}+c+d \lambda .
$$

The solution of Eq.(2.5) with the conditions (2.2) is

$$
\begin{aligned}
v(s) & =\left(\frac{a^{2}\left(e^{-a}-\lambda\right)+\left(e^{-a}-1\right)\left(b \lambda^{-1}+c+d \lambda\right)}{a^{2}\left(e^{-a}-e^{a}\right)}\right) e^{a s} \\
& +\left(\frac{a^{2}\left(\lambda-e^{a}\right)-\left(e^{a}-1\right)\left(b \lambda^{-1}+c+d \lambda\right)}{a^{2}\left(e^{-a}-e^{a}\right)}\right) e^{-a s} \\
& -\left(\frac{b \lambda^{-1}}{a^{2}}+\frac{c}{a^{2}}+\frac{d \lambda}{a^{2}}\right) .
\end{aligned}
$$

Since $x(t)=\lambda^{n} v(t-n)$, we have the following solution, denoted by $x_{n}(t)$ on the interval $[n, n+1)$

$$
\begin{aligned}
x_{n}(t) & =\frac{\lambda^{n-1} b}{a^{2}\left(e^{-a}-e^{a}\right)}\left\{e^{a(t-n-1)}-e^{-a(t-n-1)}-e^{a(t-n)}+e^{-a(t-n)}+e^{a}-e^{-a}\right\} \\
& +\frac{\lambda^{n}}{a^{2}\left(e^{-a}-e^{a}\right)}\left\{a^{2}\left(e^{a(t-n-1)}-e^{-a(t-n-1)}\right)\right. \\
& \left.+c\left(e^{a(t-n-1)}-e^{-a(t-n-1)}-e^{a(t-n)}+e^{-a(t-n)}+e^{a}-e^{-a}\right)\right\} \\
& +\frac{\lambda^{n+1}}{a^{2}\left(e^{-a}-e^{a}\right)}\left\{a^{2}\left(e^{-a(t-n)}-e^{a(t-n)}\right)\right. \\
& \left.+d\left(e^{a(t-n-1)}-e^{-a(t-n-1)}-e^{a(t-n)}+e^{-a(t-n)}+e^{a}-e^{-a}\right)\right\} .
\end{aligned}
$$

We can also extend this solution on the interval $[0, \infty)$ as follows by taking $n-1=$ $[t-1], n=[t], n+1=[t+1]$ and $t-n=t-[t]=\{t\}$ which denotes the fractional part of $t$ :

$$
\begin{aligned}
x_{\lambda}(t) & =\frac{\lambda^{[t-1]} b}{a^{2}\left(e^{-a}-e^{a}\right)}\left\{e^{a(\{t\}-1)}-e^{-a(\{t\}-1)}-e^{a\{t\}}+e^{-a\{t\}}+e^{a}-e^{-a}\right\} \\
& +\frac{\lambda^{[t]}}{a^{2}\left(e^{-a}-e^{a}\right)}\left\{a^{2}\left(e^{a(\{t\}-1)}-e^{-a(\{t\}-1)}\right)\right. \\
& \left.+c\left(e^{a(\{t\}-1)}-e^{-a(\{t\}-1)}-e^{a\{t\}}+e^{-a\{t\}}+e^{a}-e^{-a}\right)\right\} \\
& +\frac{\lambda^{[t+1]}}{a^{2}\left(e^{-a}-e^{a}\right)}\left\{a^{2}\left(e^{-a\{t\}}-e^{a\{t\}}\right)\right. \\
& \left.+d\left(e^{a(\{t\}-1)}-e^{-a(\{t\}-1)}-e^{a\{t\}}+e^{-a\{t\}}+e^{a}-e^{-a}\right)\right\} .
\end{aligned}
$$

On the other hand, from the condition (1.4) we have

$$
x_{n}^{\prime}(n+1)=(1-\alpha) x_{n+1}^{\prime}(n+1)
$$

since the first derivative of the solution is right continuous. Here $x_{n}^{\prime}(t)$ and $x_{n+1}^{\prime}(t)$ denote the derivatives of the solutions $x_{n}(t)$ and $x_{n+1}(t)$ on the intervals $[n, n+1)$ and $[n+1, n+2)$, respectively. 
Now, by taking the derivative of (2.6), we obtain

$$
\begin{aligned}
x_{n}^{\prime}(t) & =\frac{\lambda^{n-1} b}{a\left(e^{-a}-e^{a}\right)}\left\{e^{a(t-n-1)}+e^{-a(t-n-1)}-e^{a(t-n)}-e^{-a(t-n)}\right\} \\
& +\frac{\lambda^{n}}{a\left(e^{-a}-e^{a}\right)}\left\{a^{2}\left(e^{a(t-n-1)}+e^{-a(t-n-1)}\right)\right. \\
& \left.+c\left(e^{a(t-n-1)}+e^{-a(t-n-1)}-e^{a(t-n)}-e^{-a(t-n)}\right)\right\} \\
& +\frac{\lambda^{n+1}}{a\left(e^{-a}-e^{a}\right)}\left\{a^{2}\left(-e^{-a(t-n)}-e^{a(t-n)}\right)\right. \\
& \left.+d\left(e^{a(t-n-1)}+e^{-a(t-n-1)}-e^{a(t-n)}-e^{-a(t-n)}\right)\right\} .
\end{aligned}
$$

Substituting $n+1$ in place of $n$ in (2.9) and considering the equation (2.8) gives us the following equation

$$
\begin{aligned}
& \lambda^{3}+\left(\frac{\cosh a(1-\alpha)\left(a^{2}+c\right)-c(1-\alpha)+a^{2} \cosh a+d(\cosh a-1)}{(1-\alpha)\left(d(\cosh a-1)-a^{2}\right)}\right) \lambda^{2} \\
& +\left(\frac{(1-\alpha)(b+c)(\cosh a-1)-a^{2}}{(1-\alpha)\left(d(\cosh a-1)-a^{2}\right)}\right) \lambda+\frac{b(\cosh a-1)}{(1-\alpha)\left(d(\cosh a-1)-a^{2}\right)}=0
\end{aligned}
$$

which is called the characteristic equation of the Eq.(1.3)-(1.4). Eq.(2.10) has three nontrivial solutions. If $\lambda_{1}, \lambda_{2}$ and $\lambda_{3}$ are different characteristic roots, the corresponding solutions $x_{\lambda_{1}}, x_{\lambda_{2}}$ and $x_{\lambda_{3}}$, which are obtained by taking $\lambda_{1}, \lambda_{2}$ and $\lambda_{3}$ in place of $\lambda$ in the solution (2.7), are independent solutions of the Eq.(1.3)-(1.4). We can also write the general solution of Eq.(1.3)-(1.4) as

$$
x(t)=c_{1} x_{\lambda_{1}}(t)+c_{2} x_{\lambda_{2}}(t)+c_{3} x_{\lambda_{3}}(t)
$$

where $c_{i}, i=1,2,3$ are arbitrary constants.

2.2. Theorem. Consider Eq.(1.3)-(1.4) with the initial conditions

$$
x(-1)=x_{-1}, x(0)=x_{0}, x^{\prime}(0)=y_{0} .
$$

If $\lambda_{i}, i=1,2,3$, are distinct roots of Eq. (2.10) and

$$
\lambda_{2} \lambda_{3}\left(\lambda_{2}-\lambda_{3}\right)+\lambda_{1} \lambda_{2}\left(\lambda_{1}-\lambda_{2}\right)-\lambda_{1} \lambda_{3}\left(\lambda_{1}-\lambda_{3}\right) \neq 0,
$$

then the initial value problem (1.3)-(1.4) with (2.12) has a unique solution on the interval $[0, \infty)$ where $x_{-1}, x_{0}$ and $y_{0}$ are given numbers.

Proof. If $\lambda_{i}, i=1,2,3$, are distinct roots of the Eq. (2.10), then the general solution of the Eq.(1.3)-(1.4) is given by (2.11). Let us substitute the initial conditions (2.12) into (2.11), then we have

$$
\left\{\begin{array}{l}
c_{1} x_{\lambda_{1}}(-1)+c_{2} x_{\lambda_{2}}(-1)+c_{3} x_{\lambda_{3}}(-1)=x_{-1}, \\
c_{1} x_{\lambda_{1}}(0)+c_{2} x_{\lambda_{2}}(0)+c_{3} x_{\lambda_{3}}(0)=x_{0} \\
c_{1} x_{\lambda_{1}}^{\prime}(0)+c_{2} x_{\lambda_{2}}^{\prime}(0)+c_{3} x_{\lambda_{3}}^{\prime}(0)=y_{0} .
\end{array}\right.
$$

Since the determinant of the coefficients matrix of the system (2.14), which is calculated as

$$
\Delta=\left(\frac{2 a^{2}-d\left(e^{-a}+e^{a}-2\right)}{a\left(e^{-a}-e^{a}\right)}\right)\left(\frac{\lambda_{2} \lambda_{3}\left(\lambda_{2}-\lambda_{3}\right)+\lambda_{1} \lambda_{2}\left(\lambda_{1}-\lambda_{2}\right)-\lambda_{1} \lambda_{3}\left(\lambda_{1}-\lambda_{3}\right)}{\lambda_{1} \lambda_{2} \lambda_{3}}\right),
$$

is different from zero then the system (2.14) has a unique solution $c_{1}, c_{2}, c_{3}$. It should be noticed that the derivative used in this proof is right hand derivative. 
2.3. Theorem. Assume that $\lambda_{1}$ and $\lambda_{2}$ are equal (say $\left.\lambda\right)$ and $\lambda_{3}$ is a distinct root of Eq.(2.10). If

$$
\lambda^{2}+2 \lambda \lambda_{3}+\lambda_{3}^{2} \neq 0,
$$

then the initial value problem (1.3)-(1.4) with (2.12) has a unique solution on the interval $[0, \infty)$.

Proof. First of all, for this case let us start with writing the general solution of Eq.(1.3)(1.4) on $[0, \infty)$ :

A general solution of Eq.(1.3)-(1.4) when $\lambda_{1}=\lambda_{2}(=\lambda)$ and $\lambda_{3}$ is a distinct characteristic root is given by

$$
x(t)=c_{1} x_{1}(t)+c_{2} x_{2}(t)+c_{3} x_{3}(t)
$$

where $c_{i}, i=1,2,3$, are arbitrary constants and

$$
\begin{aligned}
& x_{1}(t)=A_{1}([t]) W_{1}(\{t\})+A_{2}([t]) W_{2}(\{t\})+A_{3}([t]) W_{3}(\{t\}) \\
& x_{2}(t)=[t-1] A_{1}([t]) W_{1}(\{t\})+[t] A_{2}([t]) W_{2}(\{t\})+[t+1] A_{3}([t]) W_{3}(\{t\}) \\
& x_{3}(t)=x_{\lambda_{3}}(t)
\end{aligned}
$$

with

$$
A_{1}([t])=\frac{b \lambda^{[t-1]}}{a^{2}\left(e^{-a}-e^{a}\right)}, A_{2}([t])=\frac{\lambda^{[t]}}{a^{2}\left(e^{-a}-e^{a}\right)}, A_{3}([t])=\frac{\lambda^{[t+1]}}{a^{2}\left(e^{-a}-e^{a}\right)}
$$

and

$$
\begin{aligned}
W_{1}(\{t\}) & =e^{a(\{t\}-1)}-e^{-a(\{t\}-1)}-e^{a\{t\}}+e^{-a\{t\}}+e^{a}-e^{-a}, \\
W_{2}(\{t\}) & =c\left(e^{a(\{t\}-1)}-e^{-a(\{t\}-1)}-e^{a\{t\}}+e^{-a\{t\}}+e^{a}-e^{-a}\right) \\
& +a^{2}\left(e^{a(\{t\}-1)}-e^{-a(\{t\}-1)}\right), \\
W_{3}(\{t\}) & =d\left(e^{a(\{t\}-1)}-e^{-a(\{t\}-1)}-e^{a\{t\}}+e^{-a\{t\}}+e^{a}-e^{-a}\right) \\
& +a^{2}\left(e^{a(\{t\})}-e^{-a(\{t\})}\right),
\end{aligned}
$$

where $\{t\}$ is the fractional part of $t$ and $x_{\lambda_{3}}(t)$ is the same as (2.7) provided that replacing $\lambda$ with $\lambda_{3}$.

Substituting the initial conditions (2.12) into (2.16) gives us a system similar to (2.14) and the determinant of the coefficient matrix is computed as

$$
\Delta=\left(\frac{\lambda^{2}-2 \lambda \lambda_{3}+\lambda_{3}^{2}}{\lambda \lambda_{3}}\right)\left(\frac{d\left(e^{-a}+e^{a}-2\right)-2 a^{2}}{a\left(e^{-a}-e^{a}\right)}\right)
$$

which is different from zero. So there exists a unique $c_{1}, c_{2}$ and $c_{3}$.

2.4. Theorem. Assume that all roots of Eq.(2.10) are equal (i.e. $\lambda_{1}=\lambda_{2}=\lambda_{3}=\lambda$ ). Then the initial value problem (1.3)-(1.4) with (2.12) has a unique solution on the interval $[0, \infty)$.

Proof. If $\lambda_{1}=\lambda_{2}=\lambda_{3}=\lambda$, then the general solution of the Eq.(1.3)-(1.4) is given by the formula

$$
x(t)=c_{1} x_{1}(t)+c_{2} x_{2}(t)+c_{3} x_{3}(t)
$$


where $c_{i}, i=1,2,3$, are arbitrary constants and

$$
\begin{aligned}
& x_{1}(t)=A_{1}([t]) W_{1}(\{t\})+A_{2}([t]) W_{2}(\{t\})+A_{3}([t]) W_{3}(\{t\}) \\
& x_{2}(t)=[t-1] A_{1}([t]) W_{1}(\{t\})+[t] A_{2}([t]) W_{2}(\{t\})+[t+1] A_{3}([t]) W_{3}(\{t\}) \\
& x_{3}(t)=[t-1]^{2} A_{1}([t]) W_{1}(\{t\})+[t]^{2} A_{2}([t]) W_{2}(\{t\})+[t+1]^{2} A_{3}([t]) W_{3}(\{t\})
\end{aligned}
$$

where the functions $A_{i}$ and $W_{i} i=1,2,3$, are defined as in (2.18) and (2.19), respectively. When we consider the initial conditions (2.12) in (2.20), we obtain a system similar to (2.14). The determinant of the coefficient matrix is computed as

$$
\Delta=\frac{2\left(d\left(e^{-a}+e^{a}-2\right)-2 a^{2}\right)}{a\left(e^{-a}-e^{a}\right)}
$$

which is different from zero. So, the solution is unique.

2.5. Remark. If $\alpha=0$, then the equation is reduced to just a differential equation with mixed type piecewise constant arguments which is investigated in [27]. Also the characteristic equation (2.10) is reduced to Eq.(2.4) in [27].

2.6. Corollary. If $a=0$, then

$$
x_{\lambda}(t)=\frac{b\{t\}}{2}(\{t\}-1) \lambda^{[t-1]}+\left(\frac{c\{t\}}{2}-1\right)(\{t\}-1) \lambda^{[t]}+\left(\frac{d\{t\}}{2}+1\right)(\{t\}) \lambda^{[t+1]}
$$

is a solution of Eq.(1.3)-(1.4) on the interval $[0, \infty)$ which is also a limiting case of (2.7) as $a \rightarrow 0$.

2.7. Corollary. If $b+c+d=-a^{2}$, there exists constant solutions of Eq.(1.3)-(1.4). For constant solutions we do not need any extra condition on impulse condition (1.4), because a constant solution has already satisfied this condition.

\section{Main Results}

This section contains investigation of some qualitative results for Eq.(1.3)-(1.4) such as oscillation, non-oscillation and periodicity.

3.1. Theorem. If one of the following conditions is satisfied, then Eq.(1.3)-(1.4) has oscillatory solutions:

(i) $\alpha>2, b>0, c>0, d<0, b+c>\frac{a^{2}}{(\cosh a-1)(1-\alpha)}$;

(ii) $\alpha<1, \frac{a^{2}}{(\cosh a-1)(1-\alpha)}-c<b<0, c>0,0<d<\frac{a^{2}}{(\cosh a-1)}$;

(iii) $1<\alpha<2, c<0, b<0,0<\frac{a^{2}}{(\cosh a-1)}<d, b+c>\frac{a^{2}}{(\cosh a-1)(1-\alpha)}$;

(iv) $\alpha<1, b>0, c>0, b+c<\frac{a^{2}}{(\cosh a-1)(1-\alpha)}, 0<\frac{a^{2}}{(\cosh a-1)}<d$.

Proof. A solution of Eq.(1.3)-(1.4) on the interval $[n, n+1)$ is given by (2.6). If we take $t=n$ and $t=n+1$ in (2.6), we find $x(n)=\lambda^{n}$ and $x(n+1)=\lambda^{n+1}$, respectively. So we have

$$
x(n) x(n+1)=\lambda^{2 n+1}
$$

where $\lambda$ is a root of Eq.(2.10).

On the other hand, considering the characteristic equation (2.10) as a function of $\lambda$, we get

$$
f(\lambda)=\lambda^{3}+P_{1} \lambda^{2}+P_{2} \lambda+P_{3}
$$


where

$$
\begin{aligned}
P_{1} & =\frac{\cosh a(1-\alpha)\left(a^{2}+c\right)-c(1-\alpha)+a^{2} \cosh a+d(\cosh a-1)}{(1-\alpha)\left(d(\cosh a-1)-a^{2}\right)} \\
P_{2} & =\frac{(1-\alpha)(b+c)(\cosh a-1)-a^{2}}{(1-\alpha)\left(d(\cosh a-1)-a^{2}\right)} \\
P_{3} & =\frac{b(\cosh a-1)}{(1-\alpha)\left(d(\cosh a-1)-a^{2}\right)} .
\end{aligned}
$$

For the investigation of negative characteristic root, let us consider the function

$$
f(-\lambda)=-\lambda^{3}+P_{1} \lambda^{2}-P_{2} \lambda+P_{3} .
$$

Under each condition, we find the following statements for the coefficients $P_{i}, i=1,2,3$ : For (i) and (ii),

$$
P_{1}<0, P_{2}<0 \text { and } P_{3}>0,
$$

for (iii),

$$
P_{1}<0, P_{2}>0 \text { and } P_{3}<0,
$$

and for (iv)

$$
P_{1}>0, P_{2}<0 \text { and } P_{3}>0 .
$$

So, by using the well known Descartes' rule of sign method in (3.4), we conclude that the characteristic equation (2.10) has just one negative root. Thus, from (3.1), it is easy to see that $x(n) x(n+1)<0$ and this yields the oscillatory solutions of (1.3)-(1.4).

\subsection{Theorem. If}

(i) $\alpha>2, d<0, c>0$ and $b+c<\frac{a^{2}}{(1-\alpha)(\cosh a-1)}$ or

(ii) $\alpha<1,0<d<\frac{a^{2}}{\cosh a-1}, c>0$ and $0<b<\frac{a^{2}}{(1-\alpha)(\cosh a-1)}-c$,

then there exists non-oscillatory solutions of Eq.(1.3)-(1.4).

Proof. Assume that (i) is true, then the coefficients of the characteristic equation is found as

$$
P_{1}<0, P_{2}>0, P_{3}<0 .
$$

So considering (3.2) in view of the Descartes' rule of sign method, it is concluded that the characteristic equation has at least one positive root. Thus from $(3.1), x(n) x(n+1)>0$ and this means that Eq.(1.3)-(1.4) has non-oscillatory solutions.

Under the assumption of (ii), the same inequalities are obtained with the same method.

3.3. Theorem. If one of the following conditions is satisfied, then Eq.(1.3)-(1.4) has both oscillatory and non-oscillatory solutions:

(i) $\alpha>2, b<0, c>0, d<0, c>\frac{a^{2}}{(\cosh a-1)(1-\alpha)}-b$;

(ii) $\alpha<1, b>0, c>0,0<d<\frac{a^{2}}{(\cosh a-1)}, b>\frac{a^{2}}{(\cosh a-1)(1-\alpha)}-c$;

(iii) $1<\alpha<2, b>0, c<\frac{a^{2}}{(\cosh a-1)(1-\alpha)}-b<0,0<\frac{a^{2}}{(\cosh a-1)}<d$. 
Proof. For each condition, it is easy to see that

$$
P_{1}<0, P_{2}<0, P_{3}<0
$$

where $P_{i}, i=1,2,3$ are given in (3.3). So, from Descartes' rule of sign method, we conclude that there exist just one positive root of the characteristic equation (2.10) and this root yields the non-oscillatory solutions. On the other hand, the remained two roots must be negative or complex and these roots give us that there exists oscillatory solutions.

3.4. Theorem. If

(i) $\alpha<1, c>0, d>\frac{a^{2}}{\cosh a-1}, b>\frac{a^{2}}{(\cosh a-1)(1-\alpha)}-c>0$,

(ii) $1<\alpha<2, b>0,0<d<\frac{a^{2}}{\cosh a-1}, c<\frac{a^{2}}{(\cosh a-1)(1-\alpha)}-b<0$,

is satisfied, then every solution of Eq.(1.3)-(1.4) is oscillatory.

Proof. Under each condition we obtain that

$$
P_{1}>0, P_{2}>0, P_{3}>0 \text {. }
$$

So, in view of the Descartes' rule of sign method we see that Eq.(2.10) has not any positive root. This means that all roots are negative or complex. Thus, these roots yields that every solution of Eq.(1.3)-(1.4) is oscillatory.

\subsection{Theorem. Let}

$$
P_{2}>\frac{P_{1}^{2}}{4}+\frac{2 P_{3}}{P_{1}}
$$

where $P_{i}, i=1,2,3$ are given in (3.3). If any of the following conditions is satisfied, then each solution of Eq.(1.3)-(1.4) is oscillatory:

(i) $\alpha<1, b>0, c<\frac{1}{1-\alpha}\left(\frac{-a^{2} \cosh a(2+\alpha)}{\cosh a-1}-d\right), d>\frac{a^{2}}{\cosh a-1}$;

(ii) $\alpha>2, b>0, c>0, d<0$;

(iii) $\alpha<1, b<0, c>0,-c(1-\alpha)<d<0$;

(iv) $1<\alpha<2, b<0, c<0, d>\frac{a^{2}}{\cosh a-1}$.

Proof. The characteristic equation (2.10) can be written as

$$
f(\lambda)=g(\lambda)
$$

where

$$
f(\lambda)=\lambda^{2}+P_{1} \lambda+P_{2} \text { and } g(\lambda)=\frac{-P_{3}}{\lambda} .
$$

The minimum point of the parabola $f(\lambda)$ is $\lambda_{\min }=\frac{-P_{1}}{2}$. Considering (3.5) and any of (i), (ii), (iii) and (iv), we find that $g\left(\lambda_{\min }\right)<f\left(\lambda_{\min }\right)$. Thus, it is said that the parabola $f(\lambda)$ intersects the hyperbola $g(\lambda)$ at a single point with a negative abscissa. Therefore Eq.(2.10) has no positive roots which implies that every solution of Eq.(1.3)-(1.4) is oscillatory.

3.6. Theorem. Every solution of Eq.(1.3)-(1.4) is k-periodic if and only if

$$
\lambda_{i}^{k}=1
$$

where $\lambda_{i}, i=1,2,3$ are the distinct roots of the characteristic equation of (2.10) and $k>0$ is an integer. 
Proof. Assume that Eq.(1.3)-(1.4) has a k-periodic solution. Then

$$
x_{\lambda_{i}}(t+k)=x_{\lambda_{i}}(t), i=1,2,3 .
$$

From (2.7)

$$
\begin{aligned}
& \frac{\lambda^{[t+k-1]} b}{a^{2}\left(e^{-a}-e^{a}\right)}\left\{e^{a(\{t+k\}-1)}-e^{-a(\{t+k\}-1)}-e^{a\{t+k\}}+e^{-a\{t+k\}}+e^{a}-e^{-a}\right\} \\
& +\frac{\lambda^{[t+k]}}{a^{2}\left(e^{-a}-e^{a}\right)}\left\{a^{2}\left(e^{a(\{t+k\}-1)}-e^{-a(\{t+k\}-1)}\right)\right. \\
& \left.+c\left(e^{a(\{t+k\}-1)}-e^{-a(\{t+k\}-1)}-e^{a\{t+k\}}+e^{-a\{t+k\}}+e^{a}-e^{-a}\right)\right\} \\
& +\frac{\lambda^{[t+k+1]}}{a^{2}\left(e^{-a}-e^{a}\right)}\left\{a^{2}\left(e^{-a\{t+k\}}-e^{a\{t+k\}}\right)\right. \\
& \left.+d\left(e^{a(\{t+k\}-1)}-e^{-a(\{t+k\}-1)}-e^{a\{t+k\}}+e^{-a\{t+k\}}+e^{a}-e^{-a}\right)\right\} \\
& =\frac{\lambda^{[t-1]} b}{a^{2}\left(e^{-a}-e^{a}\right)}\left\{e^{a(\{t\}-1)}-e^{-a(\{t\}-1)}-e^{a\{t\}}+e^{-a\{t\}}+e^{a}-e^{-a}\right\} \\
& +\frac{\lambda^{[t]}}{a^{2}\left(e^{-a}-e^{a}\right)}\left\{a^{2}\left(e^{a(\{t\}-1)}-e^{-a(\{t\}-1)}\right)\right. \\
& \left.+c\left(e^{a(\{t\}-1)}-e^{-a(\{t\}-1)}-e^{a\{t\}}+e^{-a\{t\}}+e^{a}-e^{-a}\right)\right\} \\
& +\frac{\lambda^{[t+1]}}{a^{2}\left(e^{-a}-e^{a}\right)}\left\{a^{2}\left(e^{-a\{t\}}-e^{a\{t\}}\right)\right. \\
& \left.+d\left(e^{a(\{t\}-1)}-e^{-a(\{t\}-1)}-e^{a\{t\}}+e^{-a\{t\}}+e^{a}-e^{-a}\right)\right\} .
\end{aligned}
$$

Since $[t+k-1]=[t-1]+k,[t+k]=[t]+k,[t+k+1]=[t+1]+k,\{t+k\}=\{t\}$,

$$
\left(\lambda_{i}^{k}-1\right) x_{\lambda_{i}}(t)=0
$$

So, we get

$$
\lambda_{i}^{k}=1
$$

Now, on the contrary assume that $\lambda_{i}^{k}=1$. Consider the solution (2.6) for $n=0$ and $n=k$, respectively as follows:

$$
\begin{aligned}
x_{0}(t) & =\frac{\lambda^{-1} b}{a^{2}\left(e^{-a}-e^{a}\right)}\left\{e^{a(t-1)}-e^{-a(t-1)}-e^{a t}+e^{-a t}+e^{a}-e^{-a}\right\} \\
& +\frac{1}{a^{2}\left(e^{-a}-e^{a}\right)}\left\{a^{2}\left(e^{a(t-1)}-e^{-a(t-1)}\right)\right. \\
& \left.+c\left(e^{a(t-1)}-e^{-a(t-1)}-e^{a t}+e^{-a t}+e^{a}-e^{-a}\right)\right\} \\
& +\frac{\lambda}{a^{2}\left(e^{-a}-e^{a}\right)}\left\{a^{2}\left(e^{-a t}-e^{a t}\right)\right. \\
& \left.+d\left(e^{a(t-1)}-e^{-a(t-1)}-e^{a t}+e^{-a t}+e^{a}-e^{-a}\right)\right\}, 0 \leq t<1,
\end{aligned}
$$


and

$$
\begin{aligned}
x_{k}(t) & =\frac{\lambda^{k-1} b}{a^{2}\left(e^{-a}-e^{a}\right)}\left\{e^{a(t-k-1)}-e^{-a(t-k-1)}-e^{a(t-k)}+e^{-a(t-k)}+e^{a}-e^{-a}\right\} \\
& +\frac{\lambda^{k}}{a^{2}\left(e^{-a}-e^{a}\right)}\left\{a^{2}\left(e^{a(t-k-1)}-e^{-a(t-k-1)}\right)\right. \\
& \left.+c\left(e^{a(t-k-1)}-e^{-a(t-k-1)}-e^{a(t-k)}+e^{-a(t-k)}+e^{a}-e^{-a}\right)\right\} \\
& +\frac{\lambda^{k+1}}{a^{2}\left(e^{-a}-e^{a}\right)}\left\{a^{2}\left(e^{-a(t-k)}-e^{a(t-k)}\right)\right. \\
& \left.+d\left(e^{a(t-k-1)}-e^{-a(t-k-1)}-e^{a(t-k)}+e^{-a(t-k)}+e^{a}-e^{-a}\right)\right\}, k \leq t<k+1 .
\end{aligned}
$$

So, we have

$$
x_{0}(t-k)=x_{k}(t), k \leq t<k+1 .
$$

Again substituting $n=1$ and $n=k+1$ in (2.6), respectively we get

$$
x_{1}(t-k)=x_{k+1}(t), k+1 \leq t<k+2 .
$$

Continuing the same way, we obtain

$$
x_{k-1}(t-k)=x_{2 k-1}(t), 2 k-1 \leq t<2 k .
$$

So it is said that $x(t)$ is k-periodic.

\subsection{Corollary. If}

$$
d=b(1-\alpha)-\frac{a^{2}(1+(2-\alpha) \cosh a)}{\cosh a-1}
$$

and

$$
c=d+\frac{\alpha\left(a^{2}+b(\cosh a-1)\right)}{(\cosh a-1)(1-\alpha)},
$$

then Eq.(1.3)-(1.4) has 3-periodic solutions.

Proof. Substituting (3.6) and (3.7) in the characteristic equation (2.10) yields that

$$
\begin{aligned}
\lambda^{3}+ & \left(\frac{(1-\alpha) a^{2}(2+(2-\alpha) \cosh a)-b(\cosh a-1)\left(1+(1-\alpha)^{2}\right)}{(1-\alpha) a^{2}(2+(2-\alpha) \cosh a)-b(1-\alpha)^{2}(\cosh a-1)}\right) \lambda^{2} \\
& +\left(\frac{(1-\alpha) a^{2}(2+(2-\alpha) \cosh a)-b(\cosh a-1)\left(1+(1-\alpha)^{2}\right)}{(1-\alpha) a^{2}(2+(2-\alpha) \cosh a)-b(1-\alpha)^{2}(\cosh a-1)}\right) \lambda \\
-\frac{b(\cosh a-1)}{(1-\alpha) a^{2}(2+(2-\alpha) \cosh a)-b(1-\alpha)^{2}(\cosh a-1)} & =0
\end{aligned}
$$

The roots of (3.8) are

$$
\lambda_{1,2}=\frac{1}{2} \pm i \frac{\sqrt{3}}{2}, \lambda_{3}=\frac{b(\cosh a-1)}{(1-\alpha) a^{2}(2+(2-\alpha) \cosh a)-b(1-\alpha)^{2}(\cosh a-1)} .
$$

Since $\lambda_{1,2}^{3}=1$, according to Theorem 3.6, there exists 3-periodic solutions of Eq.(1.3)(1.4).

\subsection{Corollary. If}

$$
c=\frac{a^{2}\left(\alpha-\left(\alpha^{2}-3 \alpha+2\right) \cosh a\right)}{(\cosh a-1)\left(\alpha^{2}-3 \alpha+2\right)}
$$


and

$$
d=b+c-\frac{\alpha a^{2}}{(1-\alpha)(\cosh a-1)}
$$

then Eq.(1.3)-(1.4) has 4-periodic solutions.

Proof. If (3.9) and (3.10) are true, then the characteristic roots of the Eq.(2.10) are found as

$$
\lambda_{1,2}= \pm i, \lambda_{3}=\frac{-b(\cosh a-1)}{(1-\alpha)\left(d(\cosh a-1)-a^{2}\right)} .
$$

Since $\lambda_{1,2}^{4}=1$, in view of Theorem 3.6, there exists 4-periodic solutions of Eq.(1.3)(1.4).

At the end of this section let us give the following examples to illustrate our results about periodicity:

3.9. Example. Consider the following equation for $t \geq 0$,

$$
\begin{aligned}
x^{\prime \prime}(t)-x(t) & =x([t-1])+\frac{3 \cosh 1}{1-\cosh 1} x([t])+\frac{\cosh 1}{1-\cosh 1} x([t+1]), t \neq n, \\
\Delta x^{\prime}(n) & =2 x^{\prime}(n), t=n, n \in \mathbb{Z}^{+} .
\end{aligned}
$$

Here, all hypotheses of Corollary 3.7 are satisfied. So it is said that Eq.(3.11)-(3.12) has 3 -periodic solutions.

Indeed, the characteristic roots of corresponding characteristic equation to (3.11)(3.12) are found as

$$
\lambda_{1,2}=\frac{1}{2} \pm i \frac{\sqrt{3}}{2}, \lambda_{3}=\frac{-1+\cosh 1}{-1-\cosh 1} .
$$

So we get $\lambda_{1,2}^{3}=1$. On the other hand, it can be easily seen from the graphics of the solution as follows:

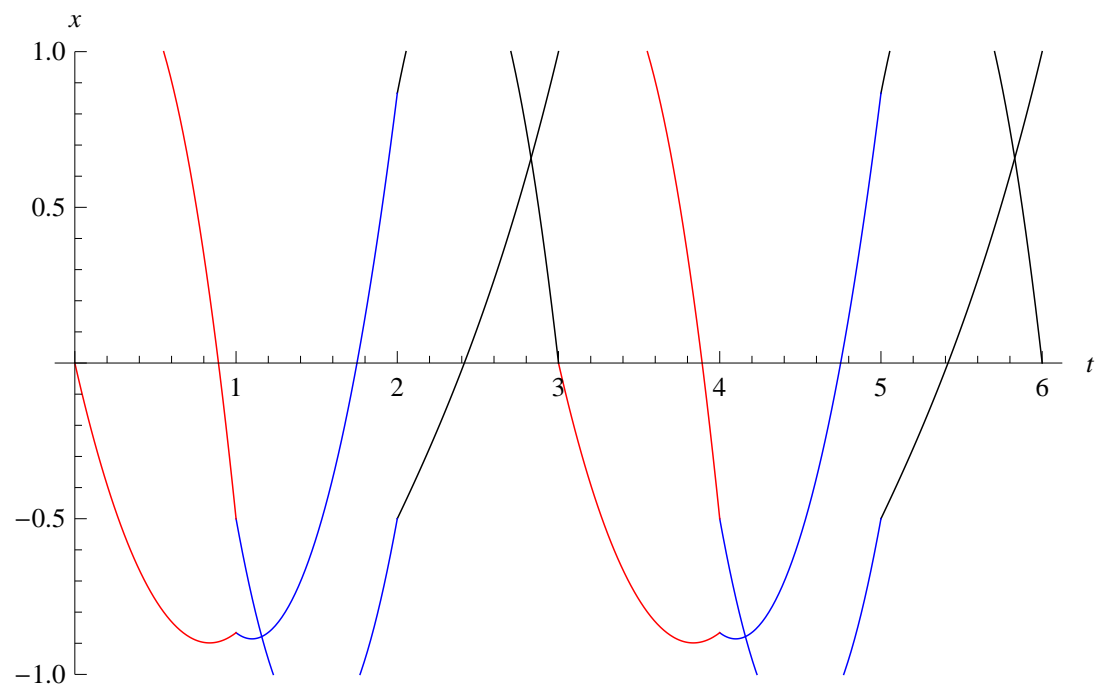

Figure 1. 3-periodic solutions of (3.11)-(3.12) 
3.10. Example. The equation

$$
\begin{aligned}
x^{\prime \prime}(t)-x(t) & =x([t-1])+\frac{3-2 \cosh 1}{2(\cosh 1-1)} x([t])+\frac{2}{\cosh 1-1} x([t+1]), t \neq n, \\
\Delta x^{\prime}(n) & =3 x^{\prime}(n), t=n, n \in \mathbb{Z}^{+} .
\end{aligned}
$$

has 4-periodic solutions because all of the conditions in Corollary 3.8 are satisfied.

The characteristic roots of corresponding characteristic equation to (3.13)-(3.14) are found as

$$
\lambda_{1,2}= \pm i, \lambda_{3}=\frac{\cosh 1-1}{2} .
$$

So we obtain $\lambda_{1,2}^{4}=1$. However, it can be easily seen from the graphics of the solution as below:

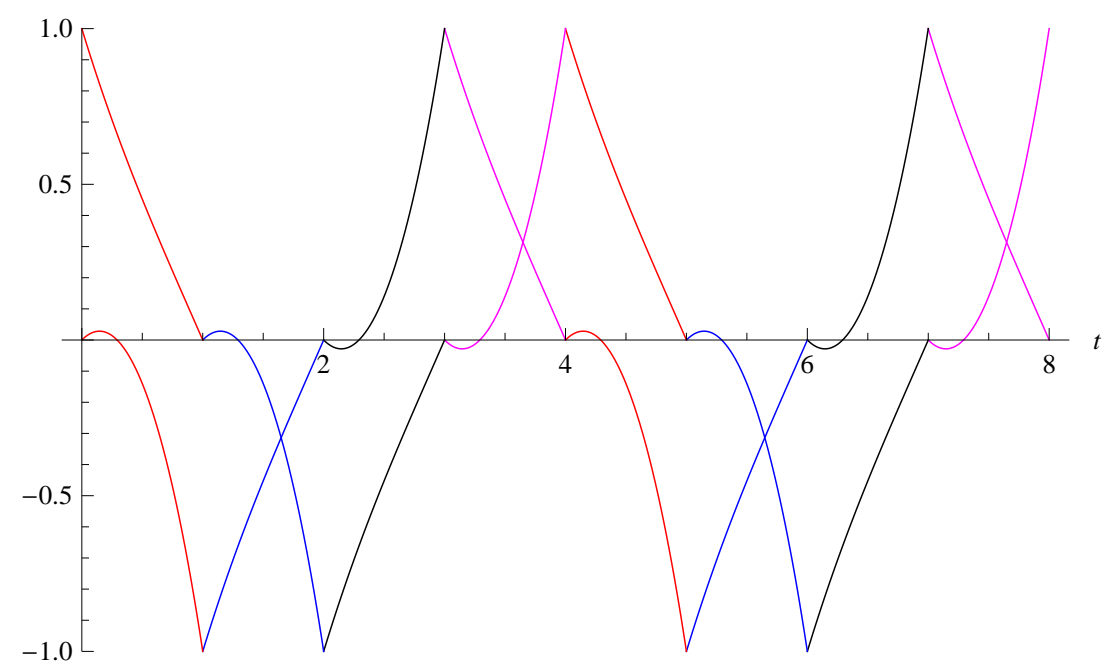

Figure 2. 4-periodic solutions of (3.13)-(3.14)

\section{References}

[1] Huang, Y.K. Oscillations and asymptotic stability of solutions of first order neutral differential equations with piecewise constant argument, The Journal of Mathematical Analysis and Applications 149 (1), 70-85, 1990.

[2] $\mathrm{Fu}, \mathrm{X}$. and $\mathrm{Li}, \mathrm{X}$. Oscillation of higher order impulsive differential equations of mixed type with constant argument at fixed time, Mathematical and Computer Modelling 48 (5), 776$786,2008$.

[3] Bereketoglu, H., Seyhan, G. and Ogun, A. Advanced impulsive differential equations with piecewise constant arguments, Mathematical Modelling and Analysis 15 (2), 175-187, 2010.

[4] Karakoc, F., Bereketoglu, H. and Seyhan, G. Oscillatory and periodic solutions of impulsive differential equations with piecewise constant argument, Acta Applicandae Mathematicae 110 (1), 499-510, 2010.

[5] Huang, C. Oscillation and nonoscillation for second order linear impulsive differential equations, The Journal of Mathematical Analysis and Applications 214 (2), 378-394, 1997.

[6] Berezansky, L. and Braverman, E. On oscillation of a second order impulsive linear delay differential equation, The Journal of Mathematical Analysis and Applications 233 (1), 276300, 1999. 
[7] Peng, M. and Ge, W. Oscillation criteria for second-order nonlinear differential equations with impulses, Computers and Mathematics with Applications 39 (5), 217-225, 2000.

[8] Yan, J. Oscillation properties of a second-order impulsive delay differential equation, Computers and Mathematics with Applications 47 (2), 253-258, 2004.

[9] Luo, Z. and Shen, J. Oscillation of second order linear differential equations with impulses, Applied Mathematics Letters 20 (1), 75-81, 2007.

[10] Ozbekler, A. and Zafer, A. Nonoscillation and oscillation of second-order impulsive differential equations with periodic coefficients, Applied Mathematics Letters 25 (3), 294-300, 2012.

[11] Ozbekler, A. and Zafer, A. Forced Oscillation of second-order impulsive differential equations with mixed nonlinearities in: Differential and Difference Equations with Applications. (Springer New York, 2013), 183-195.

[12] Leung, A. Y. T. Direct method for the steady state response of structures, The Journal of Sound and Vibration 124 (1), 135-139, 1988.

[13] Dai, L. and Singh, M. C. On oscillatory motion of spring-mass systems subjected to piecewise constant forces, The Journal of Sound and Vibration 173 (2), 217-231, 1994.

[14] Dai, L. and Singh, M. C. An analytical and numerical method for solving linear and nonlinear vibration problems, The International Journal of Solids and Structures 34 (21), 27092731, 1997.

[15] Dai, L. and Singh, M. C.Periodic, quasiperiodic and chaotic behavior of a driven Froude pendulum, The International Journal of Non-Linear Mechanics 33 (6), 947-965, 1998.

[16] Wiener, J. and Lakshmikantham, V.Excitability of a second-order delay differential equation, Nonlinear Analysis: Theory, Methods and Applications 38 (1), 1-11, 1999.

[17] Seifert, G. Second order scalar functional differential equations with piecewise constant arguments, Journal of Difference Equations and Applications 8 (5), 427-445, 2002.

[18] Chen, C.H. and Li, H.X. Almost automorphy for bounded solutions to second-order neutral differential equations with piecewise constant arguments, Electronic Journal of Differential Equations 2013 (140), 1-16, 2013.

[19] Li, H.X. Almost periodic solutions of second-order neutral delay-differential equations with piecewise constant arguments, The Journal of Mathematical Analysis and Applications 298 (2), 693-709, 2004.

[20] Yuan, R. Pseudo-almost periodic solutions of second-order neutral delay differential equations with piecewise constant argument Nonlinear Analysis: Theory, Methods and Applications 41 (7), 871-890, 2000.

[21] Yang, P., Liu, Y. and Ge, W. Green's function for second order differential equations with piecewise constant arguments Nonlinear Analysis: Theory, Methods and Applications 64 (8), 1812-1830, 2006.

[22] Nieto, J.J. and Lopez, R. R. Green's function for second-order periodic boundary value problems with piecewise constant arguments The Journal of Mathematical Analysis and Applications 304 (1), 33-57, 2005.

[23] Dads, E. A. and Lhachimi, L. New approach for the existence of pseudo almost periodic solutions for some second order differential equation with piecewise constant argument Nonlinear Analysis: Theory, Methods and Applications 64 (6), 1307-1324, 2006.

[24] Lopez, R. R. Nonlocal boundary value problems for second-order functional differential equations Nonlinear Analysis: Theory, Methods and Applications 74 (18), 7226-7239, 2011.

[25] Yuan, R. Pseudo-almost periodic solutions of second-order neutral delay differential equations with piecewise constant argument Nonlinear Analysis: Theory, Methods and Applications 41 (7), 871-890, 2000.

[26] Yuan, R. On the spectrum of almost periodic solution of second-order differential equations with piecewise constant argument Nonlinear Analysis: Theory, Methods and Applications 59 (8), 1189-1205, 2004.

[27] Bereketoglu, H., Seyhan, G. and Karakoc, F. On a second order differential equation with piecewise constant mixed arguments Carpathian Journal of Mathematics 27 (1), 1-12, 2011. 
